\title{
ASPECTOS BIOFÍSICOS DE FRUTOS E SEMENTES E EMERGÊNCIA DE PLÂNTULAS DE SANTOL
}

\footnotetext{
Noemi Vianna Martins Leão1; Marcus Victor Almeida Campos²; Sérgio Heitor Sousa Felipe ${ }^{3}$; Renata Sena Cardoso ${ }^{4}$; Elizabeth Santos Cordeiro Shimizu ${ }^{5}$

${ }^{1}$ Pesquisadora do Laboratório de Sementes Florestais, Embrapa Amazônia Oriental, Belém, Pará, Brasil. noemi.leao@embrapa.br

${ }^{2}$ Graduando do curso de Engenharia Ambiental da Universidade do Estado do Pará, Campus V, Belém, Pará, Brasil.

${ }^{3}$ Doutorando do curso de Botânica da Universidade Federal de Viçosa, Viçosa, Minas Gerais, Brasil.

${ }^{4}$ Graduanda do curso de Engenharia Florestal da Universidade Federal Rural da Amazônia, Belém, Pará, Brasil.

${ }^{5}$ Analista do Laboratório de Sementes Florestais, Embrapa Amazônia Oriental, Belém, Pará, Brasil.
}

Recebido em: 08/04/2017 - Aprovado em: 10/06/2017 - Publicado em: 20/06/2017 DOI: 10.18677/EnciBio_2017A14

\begin{abstract}
RESUMO
A biometria de sementes é uma variável de fácil medição que pode indicar a existência de variabilidade genética favorecendo a produção de mudas com elevado padrão de qualidade. O presente trabalho teve como objetivo avaliar a morfometria e massa fresca de frutos e sementes e a emergência inicial de plântulas de três matrizes de santol [Sandoricum koetjape (Burm. f.) Merr.]. Os frutos e sementes são procedentes de três árvores matrizes localizadas em Belém, estado do Pará. Para as variáveis biofísicas de frutos e sementes foram utilizados 50 e 100 amostras, respectivamente. A emergência de plântulas foi realizada em substrato areia com serragem, na proporção de 1:1, constando de quatro repetições com 25 sementes. Observou-se elevada variabilidade para massa fresca dos frutos e sementes, número de sementes por fruto, massa fresca do número de sementes por fruto. As árvores matrizes de santol apresentaram potencial para coleta de sementes a serem destinadas para produção de mudas.
\end{abstract}

PALAVRAS-CHAVE: arborização, biometria, Sandoricum koetjape

\section{BIOPHYSICAL ASPECTS OF FRUIT AND SEEDS AND EMERGENCE OF SANTOL SEEDLINGS}

\begin{abstract}
Seed biometry is an easily measured variable that may indicate an existence of genetic variation favoring the production seedlings high-quality. The present study aimed to evaluate the morphometry and fresh mass of fruits and seeds and the initial emergence of seedlings from three mother-trees of santol [Sandoricum koetjape (Burm. f.) Merr.]. The fruits were collected in three mother-trees from Belém, Pará State. For the biophysical aspects of fruits and seeds, 50 and 100 samples were
\end{abstract}


used, respectively. The emergence of seedlings was carried out on a sand substrate with sawdust, in the ratio of $1: 1$, consisting of four replicates with 25 seeds. It was observed high variability for fresh fruit mass, the number of seeds per fruit, fresh mass of the number of seeds per fruit and fresh seed mass. The santol mother trees have the potential for seed collection for the production of seedlings.

KEYWORDS: afforestation, biometry, Sandoricum koetjape

\section{INTRODUÇÃO}

A arborização urbana promove múltiplos benefícios à população (ZEM \& BATISTA, 2014; ALVES et al., 2017), pois contribui diretamente para o aumento da qualidade de vida humana através dos seguintes aspectos: (i) melhoria do microclima; (ii) redução da poluição do ar; (iii) redução do ruído; (iv) interceptação da água de chuva; (v) retenção de carbono; e (vi) valores estéticos e culturais (NICODEMO \& PRIMAVESI, 2009). Segundo AYRES (2010), espécies que propiciam sombra auxiliam na melhoria da sensação térmica do microclima, tornando o ambiente mais confortável. Nesse contexto, o santol [Sandoricum koetjape (Burm. f.) Merr.], pertencente à família Meliaceae, apresenta potencial para a arborização de parques por ter características favoráveis como a formação de copa frondosa.

A árvore de santol é típica das florestas tropicais e bastante cultivada no Sudeste Asiático (MABBERLEY et al., 1995), porém é exótica no Brasil. Essa árvore apresenta altura que varia entre 15 e 25 m e diâmetro do fuste igual ou maior a 80 $\mathrm{cm}$. Apresenta flores bissexuais com inflorescências em panículas, sendo que as folhas são trifoliadas, oblongas, elípticas ou ovais, e, de coloração verde escura. Porém, antes das flores de santol cair, a coloração muda para vermelha (QUIÑONES, 1999). O fruto é globoso e carnoso com polpa de coloração branca e aparência felpuda (GEILFUS, 1989).

S. koetjape apresenta múltiplos usos: (i) arborização urbana (MABBERLEY et al., 1995); (ii) a madeira é moderadamente dura e de fácil polimento, características que propiciam seu uso na construção de casas, barris, barcos, carrinhos e blocos de açougue (MABBERLEY et al., 1995); e (iii) os frutos (com presença de terpenos) e as sementes (com presença de limonenos) apresentam potencial para uso na medicina por possuírem propriedades anti-inflamatórias (RASADAH et al., 2004).

Estudos relacionados à biometria de sementes fornecem informações que podem ser aplicadas no manejo florestal, uma vez que a biometria é uma variável de fácil obtenção e avaliação que pode ser utilizada em diferentes espécies para indicar a existência de variabilidade entre indivíduos da mesma espécie e contribuir diretamente para a produção de mudas com maior padrão de qualidade e com base genética mais ampla (LEÃO et al., 2015; LEÃO et al., 2016). Adicionalmente, informações morfométricas de frutos e sementes podem contribuir para pesquisas de melhoramento genético (YOKOMIZO et al., 2016).

Ressaltando que a urbanização está diretamente ligada ao processo de antropização do meio ambiente natural (SCHEUER \& NEVES, 2016), as cidades são constituídas por ambientes alterados, com acentuação das condições de estresse para as plantas através da poluição urbana, as populações de árvores com baixa variabilidade genética nessas condições são mais vulneráveis ao ataque de pragas e doenças (YAMAMOTO et al., 2007).

Diante da importância de se avaliar a biometria de frutos e sementes, diversos estudos foram realizados para diferentes espécies arbóreas, como em Melanoxylon brauna (SILVA et al., 2013), Byrsonima dealbata (LOURENÇO et al., 2013), Cordia 
alliodora (MARANHO et al., 2014), Swartzia recurva (SANTOS et al., 2015), Handroanthus serratifolius (LEÃO et al., 2015), Bauhinia forficata (DUTRA et al., 2016) e Delonix regia (DUTRA et al., 2017) para observar a presença ou ausência de variabilidade.

Considerando a necessidade do desenvolvimento de maiores estudos sobre as características biofísicas relacionadas aos frutos e sementes de santol, o presente trabalho teve como objetivo avaliar os aspectos biofísicos de frutos e sementes e a emergência inicial de plântulas de três árvores matrizes de santol [Sandoricum koetjape (Burm. f.) Merr.] para verificar a variabilidade das sementes e o potencial uso para a produção de mudas.

\section{MATERIAL E MÉTODOS}

A pesquisa foi realizada no Laboratório de Sementes Florestais (LASF) da Embrapa Amazônia Oriental, Belém, PA. Os frutos de santol foram coletados no período de novembro de 2016 em três árvores matrizes localizadas em Belém, estado do Pará (matriz 1: $01^{\circ} 26^{\prime} 19,5^{\prime \prime S}$ e $48^{\circ} 26^{\prime} 46,5^{\prime \prime W O}$; matriz 2: $01^{\circ} 26^{\prime} 19,8^{\prime \prime S}$ e $48^{\circ} 26^{\prime} 47,2^{\prime \prime W O}$; e, matriz 3: $01^{\circ} 26^{\prime} 18,8^{\prime \prime S}$ e $\left.48^{\circ} 26^{\prime} 48,4^{\prime \prime} \mathrm{WO}\right)$.

Para a análise morfométrica, da massa fresca, do número de sementes por fruto e massa fresca do número de sementes por fruto foram selecionados ao acaso 50 frutos da árvore 1, 2 e 3 . O comprimento foi determinado no sentido longitudinal, e, o diâmetro foi medido no meio de cada fruto. A massa fresca de cada fruto foi realizada através da pesagem individual dos mesmos. Posteriormente, determinouse o número de sementes por fruto e a massa fresca do número de sementes por fruto.

Para a morfometria e massa das sementes foram selecionadas ao acaso 100 unidades por matriz e, posteriormente, realizaram-se as determinações do comprimento, largura e espessura. A massa fresca das sementes foi realizada conforme descrito para frutos. A biometria foi realizada utilizando paquímetro digital e a biomassa balança analítica de precisão.

Após a extração das sementes formou-se um lote com as três matrizes e foi determinado o grau de umidade das mesmas, por meio do método de estufa a $105 \pm$ $3{ }^{\circ} \mathrm{C}$, durante 24 horas, e o peso de mil sementes seguindo as recomendações das Regras para Análise de Sementes do Ministério da Agricultura, Pecuária e Abastecimento (BRASIL, 2009).

O teste de emergência foi realizado em condições de temperatura ambiente (26 ${ }^{\circ} \mathrm{C}$ ), em bandejas plásticas, constando de quatro repetições de 25 sementes, acondicionadas em substrato areia e serragem em proporções iguais. As avaliações foram realizadas diariamente e regas diárias foram realizadas quando necessário.

Foi avaliada a porcentagem de emergência ( $E \%)$, tempo médio de emergência (TME), e índice de velocidade de emergência (IVE). A porcentagem de emergência $(E \%)$ pode ser determinada pela Equação 1, em que considerou-se emergidas as sementes que apresentaram cotilédones abertos e presença de éofilos.

$$
E \%=\left(\frac{\Sigma E}{\Sigma S}\right) * 100
$$

em que

$\mathbb{Z}_{\mathrm{E}}=$ somatório de sementes emergidas até o momento de estabilização da emergência;

$\Sigma_{S}=0$ somatório de sementes colocadas para emegir 
O tempo médio de emergência (TME), foi realizado de acordo com a Equação 2, proposta por EDWARDS (1934).

$$
T M E=\frac{N 1 T 1+N 2 T 2+N 3 T 3+\cdots+N n T n}{N 1+N 2+\ldots+N n}
$$

em que,

$N_{1}, N_{2}, \ldots, N_{n}$ é o número de sementes emergidas nos tempos $T_{1}, T_{2}, \ldots T_{n}$, respectivamente;

Já o índice de velocidade de emergência (IVE) pode ser determinado pela Equação 3, de proposta por MAGUIRE (1962).

$$
I V E=\frac{E 1}{N 1}+\frac{E 2}{N 2}+\cdots+\frac{E n}{N n}
$$

em que,

$E_{1}, E_{2}, \ldots, E_{n}=$ número de sementes emergidas na primeira, segunda e última contagem;

$\mathrm{N}_{1}, \mathrm{~N}_{2}, \ldots, \mathrm{N}_{\mathrm{n}}=$ número de dias decorridos da semeadura na primeira, segunda e última contagem.

Os dados foram analisados descritivamente e, posteriormente, foi aplicado o teste de Tukey, ao nível de $5 \%(p<0,05)$. Para os dados relacionados aos frutos consideraram-se cinco repetições e a unidade experimental igual a 10 frutos, e, para as sementes cinco repetições e a unidade experimental igual a 20 sementes. $O$ software utilizado foi o ASSISTAT $7.7^{\circledR}$ (SILVA \& AZEVEDO, 2016).

\section{RESULTADOS E DISCUSSÃO}

O lote formado pelas três matrizes apresentou média de 370 sementes em um quilograma, com grau de umidade igual 45,04\%. Esse resultado pode ser aplicado no comércio de sementes para o melhor planejamento por parte do produtor. A análise do grau de umidade é essencial em testes oficiais para análise da qualidade de sementes (SARMENTO et al., 2015), devido o teor de água nas sementes influenciar diretamente a massa total das mesmas, contribuindo para um maior ou menor número de sementes por quilograma, bem como, na qualidade fisiológica.

A análise descritiva evidenciou que o comprimento dos frutos pode variar de 34,81 a $59,00 \mathrm{~mm}$, o diâmetro entre 36,28 e $68,13 \mathrm{~mm}$, massa fresca de 30,59 a $131,65 \mathrm{~g}$, e, o número de sementes por fruto de 1 a 6 unidades. Em relação ao coeficiente de assimetria, os valores foram próximos à zero para todos os parâmetros avaliados das três árvores, indicando que os dados estão próximos da distribuição normal. O coeficiente de variação para massa fresca dos frutos, número de sementes por fruto e massa fresca do número de sementes por fruto foi elevado para todas as matrizes.

Para as sementes a análise descritiva evidenciou diferentes amplitudes de tamanhos para comprimento $(20,08$ a $32,58 \mathrm{~mm})$, largura $(10,67$ a $19,63 \mathrm{~mm})$, espessura $(6,03$ a 19,98 $\mathrm{mm})$ e massa fresca $(0,63$ a $5,25 \mathrm{~g})$ ao considerar os valores mínimos e máximos das três matrizes. A assimetria foi próxima de zero, semelhante ao resultado encontrado para os frutos, exceto para espessura da 
árvore 3. Os dados relacionados à massa fresca apresentaram elevados valores de coeficiente de variação para todas as três árvores (Tabela 1).

Para outras espécies arbóreas como em Carapa guianensis (RIBEIRO et al., 2016) e Lecythis pisonis (LEÃO et al., 2016) já foi observado padrão de distribuição das variáveis biométricas e de massa de frutos e sementes próximos da normalidade, conforme os resultados do presente estudo (Tabela 1). Estudo desenvolvido por APRILIANTI \& PUTRI (2009) constatou que as sementes de santol variam em tamanho. Esses mesmos autores relatam comprimento médio igual a $24,17 \mathrm{~mm}$, largura média equivalente a $17,63 \mathrm{~mm}$, e, espessura com valor de 12,27 $\mathrm{mm}$ para o santol, corroborando com os valores encontrados nesta pesquisa.

TABELA 1. Parâmetros relacionados às características biofísicas de frutos e sementes de santol [Sandoricum koetjape (Burm. f.) Merr.] de três árvores matrizes ocorrentes no município de Belém, PA.

\begin{tabular}{|c|c|c|c|c|c|c|c|}
\hline & Parâmetro* & Mínimo & Média & Máximo & Assimetria & $\begin{array}{l}\text { Desvio } \\
\text { Padrão }\end{array}$ & $\begin{array}{l}\text { Coeficiente de } \\
\text { Variação (\%) }\end{array}$ \\
\hline & & \multicolumn{6}{|c|}{ Fruto } \\
\hline \multirow{5}{*}{ 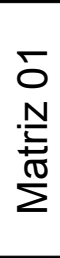 } & CF (mm) & 34,81 & 45,40 & 54,05 & $-0,15$ & 3,91 & 8,61 \\
\hline & $\mathrm{DF}(\mathrm{mm})$ & 36,28 & 53,39 & 62,66 & $-0,86$ & 5,81 & 10,88 \\
\hline & $\mathrm{MF}(\mathrm{g})$ & 30,59 & 76,82 & 116,67 & 0,16 & 18,65 & 24,28 \\
\hline & NSF & 1,00 & 3,48 & 5,00 & $-0,56$ & 0,97 & 27,99 \\
\hline & $\operatorname{MFSF}(\mathrm{g})$ & 2,31 & 10,11 & 17,55 & 0,11 & 3,54 & 35,06 \\
\hline \multirow{5}{*}{ 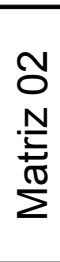 } & CF (mm) & 39,21 & 47,91 & 55,02 & $-0,27$ & 3,54 & 7,38 \\
\hline & $\mathrm{DF}(\mathrm{mm})$ & 43,97 & 55,26 & 64,75 & $-0,39$ & 5,05 & 9,14 \\
\hline & $\mathrm{MF}(\mathrm{g})$ & 40,69 & 85,59 & 120,96 & $-0,17$ & 20,50 & 23,95 \\
\hline & NSF & 2,00 & 4,26 & 5,00 & $-0,92$ & 0,88 & 20,57 \\
\hline & $\operatorname{MFSF}(\mathrm{g})$ & 6,27 & 15,97 & 27,36 & 0,11 & 4,89 & 30,61 \\
\hline \multirow{5}{*}{ 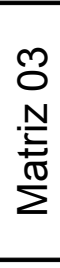 } & $\mathrm{CF}(\mathrm{mm})$ & 40,52 & 49,69 & 59,00 & 0,13 & 3,84 & 7,73 \\
\hline & $\mathrm{DF}(\mathrm{mm})$ & 48,68 & 56,78 & 68,13 & 0,34 & 4,42 & 7,79 \\
\hline & $\mathrm{MF}(\mathrm{g})$ & 56,72 & 87,20 & 131,65 & 0,37 & 19,38 & 22,23 \\
\hline & NSF & 2,00 & 4,10 & 6,00 & $-0,67$ & 1,04 & 25,25 \\
\hline & MFSF $(g)$ & 5,74 & 15,69 & 27,67 & 0,30 & 5,05 & 32,15 \\
\hline & & \multicolumn{6}{|c|}{ Semente } \\
\hline \multirow{4}{*}{ 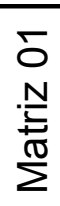 } & $\mathrm{CS}(\mathrm{mm})$ & 20,08 & 25,24 & 29,73 & $-0,14$ & 1,96 & 7,76 \\
\hline & $\mathrm{LS}(\mathrm{mm})$ & 10,67 & 14,76 & 17,49 & $-0,51$ & 1,09 & 7,41 \\
\hline & $\mathrm{ES}(\mathrm{mm})$ & 6,03 & 11,87 & 16,64 & $-0,14$ & 1,20 & 10,13 \\
\hline & MFS (g) & 0,63 & 2,15 & 2,84 & $-0,85$ & 0,38 & 17,84 \\
\hline \multirow{4}{*}{ 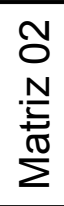 } & $\mathrm{CS}(\mathrm{mm})$ & 22,12 & 27,15 & 31,51 & $-0,26$ & 2,05 & 7,56 \\
\hline & $\mathrm{LS}(\mathrm{mm})$ & 13,58 & 16,54 & 19,63 & 0,14 & 1,92 & 7,33 \\
\hline & $\mathrm{ES}(\mathrm{mm})$ & 10,68 & 13,48 & 15,94 & $-0,17$ & 1,15 & 8,54 \\
\hline & $\operatorname{MFS}(g)$ & 1,75 & 3,19 & 5,25 & 0,19 & 0,61 & 19,05 \\
\hline \multirow{4}{*}{ 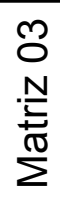 } & $\mathrm{CS}(\mathrm{mm})$ & 23,00 & 28,35 & 32,58 & $-0,26$ & 2,00 & 7,05 \\
\hline & $\mathrm{LS}(\mathrm{mm})$ & 13,75 & 16,02 & 19,35 & 0,49 & 1,13 & 7,07 \\
\hline & $\mathrm{ES}(\mathrm{mm})$ & 11,43 & 13,17 & 19,98 & 2,54 & 1,12 & 8,54 \\
\hline & MFS $(g)$ & 2,00 & 2,76 & 4,18 & 0,90 & 0,41 & 15,00 \\
\hline
\end{tabular}

${ }^{*}$ CF: comprimento do fruto; DF: diâmetro do fruto; MF: massa fresca do fruto; NSF: número de sementes por fruto; MFSF: massa fresca do número de sementes por fruto; CS: comprimento da semente; LS: largura da semente; ES: espessura da semente e MFS: massa fresca da semente. 
Árvores de Cordia ecalyculata presentes em dois tipos de ambientes contrastantes, com maior e menor nível de luminosidade natural, apresentaram diferenças biométricas para os frutos (COSSA et al., 2015). Segundo BEZERRA et al. (2014), as variações fenotípicas presentes em frutos e sementes devem-se a fatores ambientais, como solo e clima, e/ou a causas genéticas. Os elevados coeficientes de variações observado neste estudo evidenciam a presença de variabilidade para a massa fresca dos frutos, número de sementes por fruto, massa fresca do número de sementes por fruto e massa fresca das sementes (Tabela 1). As três árvores de santol apresentaram diferenças significativas entre todas as características biofísicas analisadas $(p<0,05)$ (Tabela 2 e 3$)$.

Para os frutos, o comprimento apresentou a maior média para a árvore 3 , diferindo das demais árvores, bem como, a árvore 1 teve a menor média. Em relação ao diâmetro, as árvores 2 e 3 não diferiram entre si, mas foram superiores a árvore 1. Para a massa fresca dos frutos a árvore 3 diferiu da árvore 1 , mas não diferiu da árvore 2. O número de sementes por fruto não apresentou diferenças entre as árvores 2 e 3 , porém ambas foram superiores a árvore 1. Para a massa fresca das sementes por frutos o resultado estatístico foi similar ao descrito para o número de sementes por fruto, onde as árvores 2 e 3 diferiram da árvore 1 (Tabela 2).

TABELA 2. Comparação de média entre as características biofísicas de frutos de santol [Sandoricum koetjape (Burm. f.) Merr.] de três árvores matrizes ocorrentes no município de Belém, PA.

\begin{tabular}{clllll}
\hline & \multicolumn{5}{c}{ Variáveis $^{*}$ Árvore matriz } \\
\cline { 2 - 6 } & $\mathrm{CF}(\mathrm{mm})$ & $\mathrm{DF}(\mathrm{mm})$ & $\mathrm{MF}(\mathrm{g})$ & $\mathrm{NSF}$ & $\mathrm{MFSF}(\mathrm{g})$ \\
\hline 1 & $45,40 \mathrm{c}$ & $53,39 \mathrm{~b}$ & $76,82 \mathrm{~b}$ & $3,48 \mathrm{~b}$ & $10,11 \mathrm{~b}$ \\
2 & $47,91 \mathrm{~b}$ & $55,26 \mathrm{a}$ & $85,59 \mathrm{ab}$ & $4,26 \mathrm{a}$ & $15,97 \mathrm{a}$ \\
3 & $49,69 \mathrm{a}$ & $56,78 \mathrm{a}$ & $87,20 \mathrm{a}$ & $4,10 \mathrm{a}$ & $15,69 \mathrm{a}$ \\
\hline $\mathrm{CV}(\%)$ & 1,83 & 1,75 & 7,10 & 7,53 & 13,31 \\
\hline
\end{tabular}

* $\mathrm{CF}$ : comprimento do fruto; DF: diâmetro do fruto; MF: massa fresca do fruto; NSF: número de sementes por fruto; MFSF: massa fresca do número de sementes por fruto.

Para as sementes, o comprimento exibiu maior média para a árvore 3 , sendo superior as demais árvores, e, a árvore 1 apresentou a menor média. Em relação à largura, as árvores 2 e 3 não diferiram entre si, mas ambas foram maiores, em comparação a árvore 1. Para a espessura o resultado foi semelhante ao observado para largura das sementes, não havendo diferenças entre as árvores 2 e 3, mas ambas foram superiores a árvore 1. Quanto a massa fresca, a árvore 2 apresentou maior média em relação as árvores 1 e 3 (Tabela 3).

TABELA 3. Comparação de média entre as características biofísicas de sementes de santol [Sandoricum koetjape (Burm. f.) Merr.] de três árvores matrizes ocorrentes no município de Belém, PA.

\begin{tabular}{cllll}
\hline & \multicolumn{4}{c}{ Variáveis $^{*}$} \\
\cline { 2 - 5 } Árvore matriz & $\mathrm{CS}(\mathrm{mm})$ & LS $(\mathrm{mm})$ & $\mathrm{ES}(\mathrm{mm})$ & $\mathrm{MFS}(\mathrm{g})$ \\
\hline 1 & $25,24 \mathrm{c}$ & $14,76 \mathrm{~b}$ & $11,87 \mathrm{~b}$ & $2,15 \mathrm{c}$ \\
2 & $27,15 \mathrm{~b}$ & $16,54 \mathrm{a}$ & $13,48 \mathrm{a}$ & $3,19 \mathrm{a}$ \\
3 & $28,35 \mathrm{a}$ & $16,02 \mathrm{a}$ & $13,17 \mathrm{a}$ & $2,76 \mathrm{~b}$ \\
\hline $\mathrm{CV}(\%)$ & 2,24 & 2,10 & 2,11 & 5,61 \\
\hline
\end{tabular}


${ }^{*}$ CS: comprimento da semente; LS: largura da semente; ES:

espessura da semente e MFS: massa fresca da semente.

Em diferentes espécies como em Cassia fistula (BEZERRA et al., 2014), Ormosia arborea (SILVA et al., 2014), Albizia edwallii (DUARTE et al., 2015), Acacia farnesiana (VASCONCELOS et al., 2015), Apuleia molaris (REIS et al., 2016), Poincianella pyramidalis (MENDONÇA et al., 2016), Hymenaea courbaril (SOUZA \& SEGATO, 2016; DUARTE et al., 2016), Balfourodendron riedelianum (GOMES et al., 2016), Caesalpinia ferrea (ANDRADE et al., 2016), Senna multijuga (SILVA et al., 2016), Delonix regia (DUTRA et al., 2017) e Ceiba speciosa (ROVERI NETO \& DE PAULA, 2017) foi demonstrada variações biométricas das sementes, conforme os dados desta pesquisa.

Recentemente, estudo realizado por ZUCHI et al. (2016), ao avaliarem três estratos de sementes com base no comprimento (sementes curtas, intermediárias e longas), observaram que as sementes longas apresentaram maior velocidade de emergência e crescimento de plantas. No presente estudo a porcentagem, o tempo médio e o índice de velocidade de emergência não apresentaram diferenças significativas entre as árvores $(p \geq 0,05)$, embora as sementes da árvore matriz 1 sejam menores em relação a matriz 2 e 3 (Tabela 3 ).

No geral, as árvores 1, 2 e 3 apresentaram elevada média de porcentagem de emergência, com valores iguais a 85,97 e $82 \%$, respectivamente. O tempo médio de emergência variou entre 25,71 e 26,31 dias e o índice de velocidade de emergência entre 0,81 e 0,95 . Os resultados de porcentagem de emergência do presente estudo estão de acordo com o observado por APRILIANTI \& PUTRI (2009) que encontraram valores de $95 \%$ de emergência para sementes de santol com tempo de armazenamento até 30 dias. Para Swietenia macrophylla, espécie pertencente à família Meliaceae, também já foi relatada elevada porcentagem de emergência (CARVALHO et al., 2016).

\section{CONCLUSÃO}

Há elevada variabilidade para massa fresca dos frutos e sementes, número de sementes por fruto e massa fresca do número de sementes por fruto de santol.

As árvores matrizes de santol apresentam potencial para coleta de sementes a serem destinadas para produção de mudas.

\section{REFERÊNCIAS}

ALVES, A.K.; JESUS, A.T.C.; MACÊDO, K.C.; SAMPAIO, M.D.M.A. Avaliação do conforto urbano sob a influência da vegetação na cidade de Cuiabá-MT. E\&S Engineering and Science, v.6, n.1, p.106-121, 2017. Disponível em: http://periodicoscientificos.ufmt.br/ojs/index.php/eng/article/view/4757/3193.

ANDRADE, L.I.F.; LIMA, J.S.S.; OLIVEIRA, K.J.B.; SILVA SOARES, A.P.; SILVA, J.N. Biometria de vagens e viabilidade de sementes de jucá (Caesalpinia ferrea M.) no semiárido. Agropecuária Científica no Semiárido, v.12, n.1, p.73-82, 2016. Disponível em: http://revistas.ufcg.edu.br/acsa/index.php/ACSA/article/view/708/pdf.

APRILIANTI, P.; PUTRI, W.U. Studi sifat fisik biji kecapi (Sandoricum koetjape Burm. f. Merr) dan penyimpanannya dalam suhu kamar. Buletin Kebun Raya, v.12, n.2, p.61-68, $2009 . \quad$ Disponível http://jurnal.krbogor.lipi.go.id/index.php/buletin/article/view/12_2_4. 
AYRES, M.C.R. Avaliação do sombreamento natural do flamboyant (Delonix regia) na temperatura de um ambiente. Revista Agrarian, v.3, n.9, p.200-208, 2010. Disponível em: http://ojs.ufgd.edu.br/index.php? journal=agrarian\&page=article\&op=view\&path\%5B\%5D=1105.

BEZERRA, F.T.C.; ANDRADE, L.A.; BEZERRA, M.A.F.; SILVA, M.L.M.; NUNES, R.C.R.; COSTA, E.G. Biometria de frutos e sementes e tratamentos pré-germinativos em Cassia fistula L. (Fabaceae-Caesalpinioideae). Semina: Ciências Agrárias, v.35, n.4, p.2273-2285, 2014. Disponível em: http://dx.doi.org/10.5433/16790359.2012v33n6Supl1p2863. doi: 10.5433/1679-0359.2012v33n6Supl1p2863

BRASIL. Ministério da Agricultura, Pecuária e Abastecimento. Regras para análise de sementes. Brasília: MAPA/ACS, Secretaria de Defesa Agropecuária, 2009. 395p. Disponível em: http://www.agricultura.gov.br/animal/laboratorios/publicacoes.

CARVALHO, C.A.; SILVA, J.B.; ALVES, C.Z. Envelhecimento acelerado em sementes de mogno. Revista Ciência Agronômica, v.47, n.4, p.691-699, 2016. Disponível em: http://dx.doi.org/10.5935/1806-6690.20160083. doi: 10.5935/18066690.20160083

COSSA, C.A.; LIMA, C.B.; FONSECA SORACE, M.A.; JANANI, J.K.; NEGRELLE, R.R.B. Light condition on the biometry and germination of dispersal units of Cordia ecalyculata. Applied Research \& Agrotechnology, v.8, n.2, p.17-24, 2015. Disponível em: http://revistas.unicentro.br/index.php/repaa/article/view/3219/2901.

DUARTE, M.M.; FREITAS MILANI, J.E.; BLUM, C.T.; NOGUEIRA, A.C. Germinação e morfologia de sementes e plântulas de Albizia edwallii (Hoehne) Barneby \& J. W. Grimes. Revista Caatinga, v.28, n.3, p.166-173, 2015. Disponível em: http://dx.doi.org/10.1590/1983-21252015v28n319rc.. doi: 10.1590/198321252015v28n319rc.

DUARTE, M.M.; PAULA, S.R.P.D.; FERREIRA, F.R.D.L.; NOGUEIRA, A.C. Morphological characterization of fruit, seed and seedling and germination of Hymenaea courbaril L. (Fabaceae) ('Jatobá'). Journal of Seed Science, v.38, n.3, p.204-211, 2016. Disponível em: http://dx.doi.org/10.1590/2317-1545v38n3159734. doi: 10.1590/2317-1545v38n3159734

DUTRA, F.V.; CARDOSO, A.D.; SOUZA, B.A.M.; SILVA BANDEIRA, A.; MORAIS, O.M. Análise biométrica de frutos e sementes de Bauhinia forficata L. Revista Verde de Agroecologia e Desenvolvimento Sustentável, v.11, n.3, p.08-12, 2016. Disponível em: http://dx.doi.org/10.18378/rvads.v11i3.4231. doi: 10.18378/rvads.v11i3.4231

DUTRA, F.V.; CARDOSO, A.D.; SILVA BANDEIRA, A.; SILVA, R.M.; MORAIS, O.M.; PRATES, C.J.N. Biometrical characteristics of fruits and seeds of flamboyant. Scientia Agraria Paranaensis, v.16, n.1, p.127-132, 2017. Disponível em: http://erevista.unioeste.br/index.php/scientiaagraria/article/view/13987/11239. 
EDWARDS, T.I. Relations of germinations soybeans to temperature and length of incubations time. Plant Physiology, v.9, n.1, p.1-30, 1934. http://www.ncbi.nlm.nih.gov/pmc/articles/PMC439825/pdf/plntphys00319-0008.pdf.

GEILFUS, F. El árbol al servicio del agricultor: manual de agroforestería para el desarrollo rural, principios y técnicas (Vol. 1, Principios y Técnicas). Santo Domingo, RD: Enda-Caribe-Catie. 1989, 657p. Disponível em: http://orton.catie.ac.cr/repdoc/A4035e/A403501e.pdf.

GOMES, D.R.; ARAUJO, M.M.; NUNES, U.R.; AIMI, S.C. Biometry and germination of Balfourodendron riedelianum Eng. Eng. Journal of Seed Science, v.38, n.3, p.187-194, 2016. Disponível em: http://dx.doi.org/10.1590/2317-1545v38n3159311. doi: 10.1590/2317-1545v38n3159311

LEÃO, N.V.M.; FELIPE, S.H.S.; SHIMIZU, E.S.C.; DOS SANTOS FILHO, B.G.; KATO, O.R.; BENCHIMOL, R. L. Biometria e diversidade de temperaturas e substratos para a viabilidade de sementes de ipê amarelo. Informativo ABRATES, v.25, n.1, p.50-54, 2015. Disponível em: http://www.abrates.org.br/informativoabrates/edicao/18.

LEÃO, N.V.M.; ARAÚJO, E.A.A.; SHIMIZU, E.S.C.; FELIPE, S.H.S. Características biométricas e massa de frutos e sementes de Lecythis pisonis Cambess. Enciclopédia Biosfera , Centro Científico Conhecer - Goiânia, v.13, n.24, p.167175, 2016.

Disponível

em:

http://www.conhecer.org.br/enciclop/2016b/agrarias/caracteristicas

\%20biometricas.pdf. doi: 10.18677/EnciBio_2016B_015

LOURENÇO, I.P.; FIGUEIREDO, R.W.; ALVES, R.E.; ARAGÃO, F.A.; MOURA, C.F. Caracterização de frutos de genótipos de muricizeiros cultivados no litoral cearense. Revista Ciência Agronômica, v.44, n.3, p.499-504, 2013. Disponível em: http://dx.doi.org/10.1590/S1806-66902013000300011. doi: 10.1590/S180666902013000300011

MABBERLEY, D.J.; PANNELL, C.M.; SING, A.M. Flora Malesiana: Series I. Spermatophyta Volume 12, part 1. Meliaceae. Rijksherbarium, Foundation Flora Malesiana, 1995, 407 pp.

MAGUIRE, J.D. Speed of germination-aid in selection evaluation for seedling emergence and vigour. Crop Science, v.2, p.176-199, 1962. http://dx.doi.org/10.2135/cropsci1962.0011183X000200020033x. doi: 10.2135/cropsci1962.0011183X000200020033x

MARANHO, Á.S.; SOARES, I.D.; PAIVA, A.V. Biometria de frutos-sementes e emergência de plântulas de Cordia alliodora (Ruiz \& Pav.) Cham. em diferentes substratos e profundidades de semeadura. Revista Biociências, v.20, n.1, 2014. Disponível em: http://periodicos.unitau.br/ojs2.2/index.php/biociencias/article/view/1823.

MENDONÇA, A.V.R.; FREITAS, T.A.S.D.; SOUZA, L.S.; FONSECA, M.D.S.; SOUZA, J.S. Morfologia de frutos e sementes e germinação de Poincianella 
pyramidalis (Tul.) L. P. Queiroz, comb. Nov. Ciência Florestal, v.26, n.2, p.375-387, 2016. Disponível em: http://dx.doi.org/10.5902/1980509822738.. doi: $10.5902 / 1980509822738$.

NICODEMO, M.L.F.; PRIMAVESI, O. Por que manter árvores na área urbana? Embrapa Pecuária Sudeste; 2009. 41p. (Documentos/ Embrapa Pecuária Sudeste 89). Disponível em: http://www.hympar.ufscar.br/arquivos/EMBRAPA_Documentos89.pdf.

QUIÑONES, C.A.B. Efecto de la fermentación y del ácido giberélico em la germinación del santol (Sandoricum koetjape Mert.). Tesis de Ingeniero Agrónomo, Escuela Agrícola Panamericana, El Zamorano, Honduras. 1999, 18p. Disponivel em: https://bdigital.zamorano.edu/bitstream/11036/2679/1/CPA-1999T013.pdf.

RASADAH, M.A.; KHOZIRAH, S.; AZNIE, A.A.; NIK, M.M. Anti-inflammatory agents from Sandoricum koetjape Merr. Phytomedicine, v.11, n.2-3, p.261-263, 2004. Disponível em: http://dx.doi.org/10.1078/0944-7113-00339. doi: 10.1078/0944-711300339

REIS, A.R.S.; FREITAS, A.D.D.D.; LEÃO, N.V.M.; SANTOS FILHO, B.G. Morphological aspects of fruits, seeds, and seedlings, and anatomy of seedlings of Apuleia molaris Spruce ex Benth. Journal of Seed Science, v.38, n.2, p.118-128, 2016. Disponível em: http://dx.doi.org/10.1590/2317-1545v38n2158472. doi: $10.1590 / 2317-1545 v 38 n 2158472$

RIBEIRO, R.R.; MOREIRA, W.K.O.; SILVA, A.J.C.; COSTA, J.L.P.; CONCEIÇÃO JÚNIOR, Z.F.; SILVA, R.T.L. Biometria de sementes de andirobeira em ecossistema de várzea no nordeste paraense. Global Science and Technology, v.9, n.3, p.116124, $2016 . \quad$ Disponível em: http://rv.ifgoiano.edu.br/periodicos/index.php/gst/article/view/836/520.

ROVERI NETO, A.; DE PAULA, R.C. Variabilidade entre árvores matrizes de Ceiba speciosa St. Hil para características de frutos e sementes. Revista Ciência Agronômica, v.48, n.2, p.318-327, 2017. Disponível em: http://ccarevista.ufc.br/seer/index.php/ccarevista/article/view/3862/1552.

SANTOS, M.A.; BRAGA, L.F.; NETO, R.M.R.; COSTA SORATO, A.M. Aspectos morfológicos e fisiológicos da germinação e morfometria de frutos e sementes de Swartzia recurva Poe P. (Fabaceae). Ciência e Natura, v.37, n.3, p.34-54, 2015. Disponível em: http://dx.doi.org/105902/2179460X17309. doi: 105902/2179460X17309

SARMENTO, H.G.S.; SOUZA DAVID, A.M.S.; BARBOSA, M.G.; NOBRE, D.A.C.; AMARO, H.T.R. Determinação do teor de água em sementes de milho, feijão e pinhão-manso por métodos alternativos. Energia Na Agricultura, v.30, n.3, p.250256, 2015. Disponível em: http://dx.doi.org/10.17224/EnergAgric.2015v30n3p250256. doi: 10.17224/EnergAgric.2015v30n3p250-256 
SCHEUER, J.M.; NEVES, S.M.A.D.S. Planejamento urbano, áreas verdes e qualidade de vida. Revista Meio Ambiente e Sustentabilidade, v.11, n.5, p.74-89, 2016.

Disponível

em: http://www.uninter.com/web/revistameioambiente/index.php/meioAmbiente/article/vie $w / 587$.

SILVA, M.D.S.; BORGES, E.E.D.L.; LEITE, H.G.; CORTE, V.B. Biometria de frutos e sementes de Melanoxylon brauna Schott. (Fabaceae-Caesalpinioideae). Revista Cerne, v.19, n.3, p.517-524, 2013. Disponível em: http://dx.doi.org/10.1590/S010477602013000300020. doi: 10.1590/S0104-77602013000300020

SILVA, A.L.D.; DIAS, D.C.F.D.S.; LIMA, L.B.D.; MORAIS, G.A.D. Methods for overcoming seed dormancy in Ormosia arborea seeds, characterization and harvest time. Journal of Seed Science, v.36, n.3, p.318-325, 2014. Disponível em: http://dx.doi.org/10.1590/2317-1545v36n31012. doi: 10.1590/2317-1545v36n31012

SILVA, E.; GUILHERME, M.D.F.S.; SANTOS, P.S. Morphometric characterization and overcoming of dormancy by chemical agent in seeds of Senna multijuga (Rich.). Revista da Universidade Vale do Rio Verde, v.14, n.1, p.1098-1106, 2016. Disponível em: http://dx.doi.org/10.5892/ruvrd.v14i1.2916. doi: 10.5892/ruvrd.v14i1.2916

SILVA, F.D.A.S.; AZEVEDO, C.A.V. The Assistat Software Version 7.7 and its use in the analysis of experimental data. African Journal of Agricultural Research, v.11, n.39, p.3733-3740, $2016 . \quad$ Disponível em: http://www.academicjournals.org/journal/AJAR/article-abstract/5E8596460818. doi: 10.5897/AJAR2016.11522

SOUZA, V.M.S.; SEGATO, S.V. Superação de dormência em sementes de jatobá (Hymenaea courbaril L.). Nucleus, v.13, n.1, p.71-80, 2016. Disponível em: http://dx.doi.org/10.3738/1982.2278.1616. doi: 10.3738/1982.2278.1616

VASCONCELOS, M.C.; MOREIRA, F.J.C.; MESQUISTA, M.L.S.; NETO, L.P.; SOUZA, M.C.M.R. Caracterização morfobiométrica de frutos e sementes e superação da dormência em coronha (Acacia farnesiana). Revista Verde de Agroecologia e Desenvolvimento Sustentável, v.10, n.5, p.120-126, 2015. Disponível em: http://dx.doi.org/10.18378/rvads.v10i5.3612. doi: 10.18378/rvads.v10i5.3612

YAMAMOTO, M.A.; SOBIERAJSKI, G.R.; SILVA FILHO, D.F.; COUTO, H.T.Z. Árvores matrizes de Tabebuia pentaphyla (L.) hemsl. (ipê de El Salvador) e Caesalpinia pluviosa DC. (sibipiruna) em área urbana, selecionadas por meio de índice de importância. Revista da Sociedade Brasileira de Arborização Urbana, v.2, n.3, p.13-31, 2007. Disponível em: http://www.revsbau.esalq.usp.br/artigos_cientificos/artigo24.pdf.

YOKOMIZO, G.K.I.; MOCHIUTTI, S.; QUEIROZ, J.A.L.D.; SANTOS, G.R.D.; FURTADO, R.G.; BRANDÃO, A.P.; COLARES, I.B. Estimativas de parâmetros genéticos para caracteres de frutos em açaizeiros no Amapá. Ciência Florestal, v. 
26, n. 3, p.985-993, 2016. Disponível em: http://dx.doi.org/10.5902/1980509824227. doi: $10.5902 / 1980509824227$

ZEM, L.M.; BATISTA, D.B. Análise da percepção da população em relação ao vandalismo na arborização viária de Curitiba-PR. Revista da Sociedade Brasileira de Arborização Urbana, v.9, n.3, p.86-107, 2014. Disponível em: http://www.revsbau.esalq.usp.br/teste/ojs-2.3.7/index.php/REVSBAU/issue/view/3.

ZUCHI, J.; CAMELO, G.N.; SOUZA, M.M.V.; SANTANA, J.; FÁTIMA SALES, J. Emergência e crescimento de mudas de baru segundo o tamanho da semente. Multi-Science Journal, v.1, n.5, p.52-56, 2016. Disponível em: https://www.ifgoiano.edu.br/periodicos/index.php/multiscience/article/view/341/250. 